\title{
Risk factors for community-associated multidrug-resistant Pseudomonas aeruginosa in veterans with spinal cord injury and disorder: a retrospective cohort study
}

\author{
IO Kale ${ }^{1}$, MA Fitzpatrick ${ }^{1,2}$, KJ Suda ${ }^{1,3}$, SP Burns ${ }^{4,5}$, L Poggensee ${ }^{1}$, S Ramanathan ${ }^{1}$, R Sabzwari ${ }^{6,7}$ and \\ CT Evans ${ }^{1,8}$
}

Study design: Retrospective cohort study

Objectives: To identify independent risk factors associated with community-associated multidrug-resistant Psedomonas aeruginosa (MDRPA) in a population of veterans with spinal cord injury and disorders (SCI/D).

Setting: A total of 127 Veterans Affairs healthcare facilities.

Methods: Laboratory results from 1 January 2012 to 31 December 2013 were collected, and MDRPA cultures were compared with non-MDRPA cultures.

Results: One thousand four hundred forty-one cultures were collected from Veterans with SCI/D, including 227 cultures with MDRPA isolates. Characteristics associated with an increased odds of MDRPA include age 50-64 (adjusted odds ratio (aOR) $=1.80,95 \%$ confidence interval $(\mathrm{Cl})=1.13-2.87)$, MDRPA culture in the past 365 days $(\mathrm{aOR}=9.12,95 \% \mathrm{Cl}=5.88-14.15)$ and carbapenem exposure in the past 90 days $(\mathrm{aOR}=2.56,95 \% \mathrm{Cl}=1.35-4.87)$. In contrast, paraplegia was associated with a $53 \%$ decreased odds of MDRPA compared with those with tetraplegia ( $a \mathrm{OR}=0.47,95 \% \mathrm{Cl}=0.32-0.69)$.

Conclusions: Risk factors for community-associated MDRPA include prior history of MDRPA and exposure to carbapenems. Awareness of these factors is important for targeted prevention and treatment of MDRPA in patients with SCI/D.

Spinal Cord (2017) 55, 687-691; doi:10.1038/sc.2017.7; published online 7 February 2017

\section{INTRODUCTION}

Pseudomonas aeruginosa is a common gram-negative organism that was responsible for $8 \%$ of all healthcare-associated infections (HAIs) in 2013. ${ }^{1}$ Antibiotic resistance in $P$. aeruginosa is an increasingly serious threat in multiple healthcare settings ${ }^{2}$ as there are a decreasing number of effective drugs to combat the bacteria. Multidrug-resistant strains account for $13-18.5 \%$ of healthcare-associated $P$. aeruginosa infections ${ }^{1,3}$ and $\sim 440$ deaths per year in the US. ${ }^{1}$ This growing level of drug resistance is a result of the spread of resistant strains from patient to patient as well as newly acquired resistance due to previous antibiotic exposure. ${ }^{4}$

Several studies have identified risk factors for acquiring multidrugresistant $P$. aeruginosa (MDRPA) in the general patient population. These include advanced age, severe injury (bedridden), use of invasive devices, admission from a chronic care facility, hospital unit and treatment with inappropriate antibiotic therapy or a number of antibiotic classes., ${ }^{3,5,6}$ In particular, hospital location and site of infection appear to substantially influence the risk for MDRPA. De Oliveria Costa et al. found that longer time to adequate antibiotic therapy was associated with greater risk of infection by MDR gram- negative bacteria among patients in the pediatric oncology intensive care unit (ICU). ${ }^{3}$ According to the National Healthcare Safety Network, $16.8 \%$ of catheter-associated bloodstream infections are attributed to MDRPA in the ICU compared with $13.3 \%$ in non-ICU areas, whereas $12.6 \%$ of catheter-associated urinary tract infection occurs from MDRPA in the ICU, as opposed to the $15.6 \%$ found in non-ICU areas. $^{2}$

Many studies of MDRPA have focused on HAIs; however, there is increasing antibiotic resistance observed in gram-negative bacteria isolated from patients in the community. ${ }^{7}$ About $30 \%$ of all infections by Pseudomonas species are community associated. ${ }^{8}$ Most of the published literature regarding community-associated $P$. aeruginosa infections is comprised of case reports and case series. Although rare, these infections can occur in previously healthy individuals and can cause pneumonia that is rapidly progressive and fatal. ${ }^{9-11}$

Patients with spinal cord injury and disorders (SCI/D) are at particularly high risk of HAIs and infection with MDR pathogens due to functional impairments, comorbidities, frequent use of invasive devices and frequent antibiotic and healthcare exposures. ${ }^{12-14}$ Characteristics unique to SCI/D patients, such as completeness of

\footnotetext{
${ }^{1}$ Department of Veterans Affairs, Center of Innovation for Complex Chronic Healthcare, Edward Hines Jr VA Hospital, Hines, IL, USA; ${ }^{2}$ Department of Medicine, Division of Infectious Diseases, Loyola University Stritch School of Medicine, Maywood, IL, USA; ${ }^{3}$ Department of Pharmacy Systems, Outcomes and Policy, College of Pharmacy, University of Illinois at Chicago, Chicago, IL, USA; ${ }^{4}$ Department of Veterans Affairs, VA Puget Sound Healthcare System, Seattle, WA, USA; ${ }^{5}$ Department of Rehabilitation Medicine, University of Washington, Seattle, WA, USA; ${ }^{6}$ Infectious Disease, Edward Hines Jr VA Hospital, Hines, IL, USA; ${ }^{7}$ Loyola University Medical Center, Maywood, IL, USA and ${ }^{8}$ Center for Healthcare Studies and Department of Preventive Medicine Institute for Public Health and Medicine, Northwestern University Feinberg School of Medicine, Chicago, IL, USA Correspondence: IO Kale, Department of Veterans Affairs, Center of Innovation for Complex Chronic Healthcare, Edward Hines Jr VA Hospital, 5000 S 5th Avenue (151H), Hines, IL 60141, USA.

E-mail: Ibuola.Kale@va.gov

Received 24 August 2016; revised 1 December 2016; accepted 30 December 2016; published online 7 February 2017
} 
Table 1 Bivariate analysis of risk factors for community-associated MDRPA and non-MDRPA

\begin{tabular}{ccc}
\hline Frequency $(\%)^{\mathrm{A}}$ & OR $(95 \% \mathrm{Cl})$ \\
\cline { 2 - 3 } & MDRPA & Non-MDRPA \\
$\mathrm{N}=227$ & $\mathrm{~N}=1214$ & \\
\hline
\end{tabular}

\section{Demographics \\ Age \\ 18-49 years \\ 50-64 years \\ $\geqslant 65$ years \\ Sex, female \\ Region \\ Northeast \\ Midwest \\ South \\ West \\ Other ${ }^{b}$}

\section{SCI/D characteristic \\ SCI/D level \\ Tetraplegia \\ Paraplegia \\ Missing \\ SCI/D onset \\ Non-traumatic \\ Traumatic \\ Missing \\ SCI/D extent \\ Incomplete \\ Complete \\ Missing \\ Duration of injury \\ 0-10 years \\ 11-20 years \\ $21+$ years \\ Missing}

Patient seen at SCI/D center

Comorbidities

Charlson comorbidity index,

median (s.d.)

Gastrostomy/jejunostomy

Renal disease

Liver disease

AIDS

Cancer/tumor

$\mathrm{CHF}$

Diabetes

CVA

Arthritis

ASPVD

COPD

Myocardial infarction

Pressure ulcer

Specimen characteristics

Specimen type

Urine
Blood
Other $^{d}$

$\begin{array}{ccc}34(15.0) & 221(18.2) & \text { Reference } \\ 104(45.8) & 469(38.6) & 1.44(0.95-2.19) \\ 89(39.2) & 524(43.2) & 1.10(0.72-1.69) \\ 7(3.1) & 34(2.8) & 1.11(0.48-2.52) \\ & & \\ 17(7.5) & 67(5.5) & \text { Reference } \\ 38(16.7) & 229(18.9) & 0.65(0.35-1.23) \\ 130(57.3) & 636(52.4) & 0.81(0.46-1.42) \\ 130(57.3) & 262(21.6) & 0.54(0.29-1.02) \\ 6(2.6) & 20(1.7) & 1.18(0.41-3.40)\end{array}$

$152(67.0) \quad 636(52.4)$ 49 (21.6) $503(44.2)$

26 (11.5) $\quad 75(6.18)$

Reference

$0.41(0.29-0.57)$

$1.45(0.90-2.34)$

40 (17.6) 259 (21.3) $\quad$ Reference

$158(69.6) \quad 860(70.8) \quad 1.19(0.82-1.73)$

$29(12.8) \quad 95(7.8) \quad 1.98(1.16-3.37)$

88 (38.8) $\quad 480(39.5)) \quad$ Reference

$94(41.4) \quad 606(49.9) \quad 0.85(0.62-1.16)$

$45(19.8) \quad 128(10.5) \quad 1.92(1.27-2.89)$

67 (29.5) $\quad 409$ (33.7) $\quad$ Reference

$42(18.5) \quad 195(16.1) \quad 1.31(0.86-2.00)$

$88(38.8) \quad 521(42.9) \quad 1.03(0.73-1.45)$

$30(13.2) \quad 89(7.3) \quad 2.06(1.26-3.35)$

$135(59.5) \quad 794(65.4) \quad 0.78(0.58-1.04)$

$\begin{array}{ccc}2(1.5) & 2(1.7) & P=0.01^{\mathrm{c}} \\ & & \\ 6(2.6) & 17(1.4) & 1.91(0.75-4.90) \\ 15(6.6) & 54(4.5) & 1.52(0.84-2.74) \\ 8(3.5) & 38(3.1) & 1.13(0.52-2.46) \\ 1(0.4) & 4(0.3) & 1.34(0.15-12.03) \\ 9(4.0) & 33(2.7) & 1.48(0.70-3.13) \\ 11(4.9) & 42(3.5) & 1.42(0.72-2.80) \\ 35(15.4) & 180(14.8) & 1.05(0.71-1.55) \\ 8(3.5) & 34(2.8) & 1.27(0.58-2.78) \\ 2(0.9) & 4(0.3) & 2.69(0.49-14.77) \\ 5(2.2) & 25(2.1) & 1.07(0.41-2.83) \\ 20(8.8) & 91(7.5) & 1.19(0.72-1.98) \\ 1(0.4) & 8(0.7) & 0.67(0.08-5.36) \\ 78(34.4) & 345(28.4) & 1.32(0.98-1.78)\end{array}$

$188(82.8) \quad 984(81.1)$

$1(0.40 \quad 11(0.9)$

38 (16.7) $\quad 219(18.0)$

Reference

$0.48(0.06-3.71)$

$0.91(0.62-1.33)$

$106(46.7) \quad 380(31.3) \quad 1.93(1.44-2.56)$
Table 1 (Continued)

\begin{tabular}{ccc}
\hline Frequency $(\%)^{\mathrm{a}}$ & OR $(95 \% \mathrm{Cl})$ \\
\cline { 2 - 3 } & MDRPA & Non-MDRPA \\
$\mathrm{N}=227$ & $\mathrm{~N}=1214$
\end{tabular}

Previous $P$. aeruginosa

culture in past 365 days

Previous MDR P. aeruginosa 73 (32.2) $\quad 42$ (3.5) $13.23(8.73-20.03)$

culture in past 365 days

Healthcare exposures in past 90 days

$\begin{array}{lccc}\text { Hospital admission } & 55(24.2) & 211(17.4) & 1.52(1.08-2.13) \\ \text { Long-term care stay } & 3(1.3) & 8(0.7) & 2.02(0.53-7.67) \\ \text { Surgery } & 8(3.5) & 42(3.5) & 1.02(0.47-2.20) \\ \text { Genitourinary procedure } & 10(4.4) & 27(2.2) & 2.03(0.97-4.25) \\ \text { Intensive care unit } & 13(5.7) & 30(2.5) & 2.40(1.23-4.57) \\ \text { Mechanical ventilation } & 16(7.1) & 28(2.3) & 3.21(1.71-6.04)\end{array}$

Medication exposure in past 90 days

$166(73.1) \quad 777(64.0) \quad 1.53(1.12-2.10)$

$\begin{array}{llll}\text { Chronic steroids }^{f} & 2(0.9) & 12(1.0) & 0.89(0.20-4.01)\end{array}$

$\begin{array}{lll}\text { Pen-G/Pen-amino } & 27(11.9) \quad 179(14.7) \quad 0.78(0.51-1.20)\end{array}$

Antistaphylococcal penicillins $\quad 0(0) \quad 3(0.25) \quad 0.76(0.04-14.79)$

Extended spectrum penicillins $23(10.1) \quad 68(5.6) \quad 1.90(1.16-3.12)$

First- and second-generation $23(10.1) \quad 97(8.0) \quad 1.30(0.81-2.10)$

cephalosporins

$\begin{array}{llll}\text { Third generation } & 26(11.5) & 80(6.6) & 1.83(1.15-2.93)\end{array}$

cephalosporins

Fourth-generation $\quad 12(5.3) \quad 29(2.4) \quad \mathbf{2 . 2 8}(\mathbf{1 . 1 5}-\mathbf{4 . 5 4})$

cephalosporins

$\begin{array}{llll}\text { Carbapenems } & 19(8.4) & 32(2.6) & \mathbf{3 . 3 8}(\mathbf{1 . 8 8}-\mathbf{6 . 0 7})\end{array}$

Macrolides $\quad 5(2.2) \quad 37(3.1) \quad 0.71(0.28-1.84)$

$\begin{array}{llll}\text { Tetracyclines } & 14(6.2) & 81(6.7) & 0.92(0.51-1.65)\end{array}$

Aminoglycosides $\quad 21(9.3) \quad 63(5.2) \quad 1.86(1.11-3.12)$

Lincomycins/clindamycin $\quad 9(4.0) \quad 24(2.0) \quad 2.05(0.94-4.47)$

Quinolones $\quad 83(36.6) \quad 305(25.1) \quad 1.72(\mathbf{1 . 2 7}-\mathbf{2 . 3 2})$

Nitrofurantoins $\quad 28(12.3) \quad 156(12.8) \quad 0.96(0.62-1.47)$

Sulfonamides $\quad 30(13.2) \quad 135(11.1) \quad 1.22(0.80-1.86)$

Colistimethate $\quad 6(2.6) \quad 2(0.2) \quad 16.47(3.30-82.11)$

$\begin{array}{llll}\text { Daptomycin } & 4(1.8) & 11(0.9) & 1.96(0.62-6.22)\end{array}$

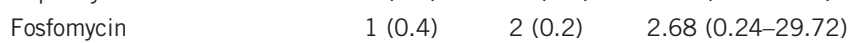

Linezolid $\quad 8(3.5) \quad 22(1.8) \quad 1.98(0.87-4.51)$

Metronidazole $\quad 15(6.6) \quad 43(3.5) \quad 1.93(1.05-3.53)$

Vancomycin $\quad 35(15.4) \quad 101(8.3) \quad 2.01(1.33-3.04)$

Abbreviations: AIDS, acquired immune deficiency syndrome; ASPVD, atherosclerotic peripheral vascular disease; $\mathrm{CHF}$, congestive heart failure; $\mathrm{Cl}$, confidence interval; $\mathrm{COPD}$, chronic obstructive pulmonary disease; CVA, cerebrovascular accident; MDR, multidrug-resistant; MDRPA; multidrug-resistant Pseudomonas aeruginosa; OR, odds ratio; SCI/D, spinal cord injury and disorder.

${ }^{\mathrm{a} A l l}$ data displayed are number (\%) unless otherwise indicated.

bIncludes San Juan, Puerto Rico, Manila and Philippines.

cWilcoxon rank sum test was used for Charlson score.

dincludes respiratory, wound, skin and stool.

eMinimally invasive or non-invasive genitourinary procedures.

fDefined as $\geqslant 85$ days of use in the prior 90 days.

injury, may influence certain infection rates. ${ }^{15} P$. aeruginosa is not an uncommon pathogen in SCI/D patients; in studies of SCI/D veterans with HAIs, P. aeruginosa accounted for $11.3-12.2 \%$ of isolated organisms. ${ }^{16,17}$ MDRPA infections in the general patient population are associated with poor clinical outcomes such as increased length of stay post infection, invasive surgery, discharge to chronic care facilities and mortality. ${ }^{5}$ However, risk factors for and clinical outcomes of 
MDRPA infections in patients with SCI/D have not been extensively studied. There are also limited data on community-associated $P$. aeruginosa infections in the SCI/D patient population. Therefore, the objective of this study was to identify independent risk factors associated with community-associated MDRPA in a population of veterans with SCI/D by comparing cultures from patients with MDRPA to those of patients with antibiotic susceptible $P$. aeruginosa.

\section{MATERIALS AND METHODS}

\section{Study setting and study design}

A retrospective cohort study was performed in veterans with SCI/D treated at any of 127 VA facilities from 1 January 2012 to 31 December 2013. The cohort included patients with a culture positive for $P$. aeruginosa, where MDRPA was defined as a $P$. aeruginosa isolate non-susceptible to a least one agent in three or more antimicrobial categories, as defined by Magiorakos, et al. ${ }^{18}$ Non-MDRPA was defined as patients with SCI/D with positive cultures of antibiotic susceptible $P$. aeruginosa. Only patients with cultures containing $P$. aeruginosa from the community were included in the study. Community-associated $P$. aeruginosa was defined as any culture obtained from a patient who (1) was seen in the outpatient setting and had no hospital admission in the previous 28 days or (2) was admitted to the hospital in the previous $48 \mathrm{~h}$ and had no previous admission in the past 28 days.

\section{Data collection}

Patient demographics, SCI/D characteristics and comorbid conditions in the past 365 days were collected from national VA data sets, including the Veterans' Health Administration Corporate Data Warehouse. The Corporate Data Warehouse is a relational database of national clinical and administrative data extracted from local electronic medical records for VA patients and is updated on a continual basis. These data sets were also used to gather information on healthcare and antibiotic exposures. Comorbid conditions were used to develop the Charlson Comorbidity Index, which was originally developed to measure mortality by weighting and summing the patient's comorbidities. A higher score indicates a greater likelihood of mortality. ${ }^{19}$ The VA SCI/D system of care includes dedicated SCI/D specialty centers called 'hubs' across the nation that provide highly specialized SCI/D care. Each of these centers connects to a variable number of 'spoke' VA facilities that provide community-based primary care for SCI/D patients. All patients were either seen at a SCI/D hub center or at a spoke facility, where care may differ. Cultures were analyzed based on these VA facilities. Geographic regions where the cultures were collected are based on the US Census regions defined by states. Locations outside the continental US, such as San Juan, Puerto Rico, Manila and Philippines were grouped into the 'other' region category.

To determine whether $P$. aeruginosa isolates were MDR, we obtained information from the Corporate Data Warehouse on all positive bacterial cultures for which antibiotic susceptibility testing was performed. These records were then filtered to include only cultures positive for P. aeruginosa. Duplicate cultures were excluded within 30 days.

\section{Statistical analysis}

Chi-square and Fisher's exact tests were used to compare categorical variables and Wilcoxon rank sum tests to compare continuous variables. Unadjusted odds ratios and 95\% confidence intervals are presented. Because some patients provided samples more than once during the study period, in both the inpatient and outpatient settings, they may have had multiple cultures positive for $P$. aeruginosa; therefore, the covariates are not entirely independent. To address this, a generalized estimating equation was used to create a multivariable logistic model that accounted for the multiple cultures taken from single patients. ${ }^{20}$ This provided risk factors and their adjusted odds ratios and $95 \%$ confidence intervals. A $P$-value $<0.05$ was considered statistically significant. Statistical analyses were conducted using SAS, version 9.3 (SAS Institute Inc., Cary, NC, USA) and Stata, version 14.1 (StataCorp LP, College Station, TX, USA).

\section{RESULTS}

A total of 5486 cultures positive for $P$. aeruginosa were collected from SCI/D patients seen at VA facilities during the study time period. Of these, $1441(26.3 \%)$ cultures were collected from 1144 patients with $P$. aeruginosa in the community setting. Four hundred ninety-six $(34.4 \%)$ patients were seen as outpatients and $945(65.6 \%)$ were admitted sometime in the $48 \mathrm{~h}$ before culture collection. Nine hundred twenty-nine $(64.5 \%)$ of the cultures were obtained from patients seen in SCI/D centers. The mean age of patients in this cohort was 61.3 years and $97.2 \%$ were male. Their median Charlson score was 2 (s.d. $=1.6)$. The most common culture site was urine $(81.3 \%)$, followed by other sites including respiratory, wound, skin and stool $(17.8 \%)$, followed by blood (0.8\%).Two hundred twenty-seven (15.6\%) of the cultures collected grew MDRPA.

Table 1 summarizes the demographic and clinical characteristics of the patients from whom the cultures were collected and displays the results of the bivariate analysis. Paraplegia was associated with lower odds of MDRPA than tetraplegia. For all other comorbidities analyzed, there were no significant differences between the two groups; however, there was a significant difference in the distribution of Charlson scores (median (s.d.); MDR: 2 (1.5); non-MDR: 2 (1.7)). Having had a previous culture positive for $P$. aeruginosa, and specifically MDR $P$. aeruginosa, in the past year was significantly associated with a positive MDRPA culture. In the 90 days before positive culture, admission to the hospital, admission to the ICU, mechanical ventilation and exposure to any antibiotic was associated with MDRPA. Exposure to a number of specific antibiotic classes was associated with increased odds of MDRPA (Table 1).

The multivariable analysis demonstrated that the level of injury, a previous MDRPA culture in the past year and exposure to carbapenems in the previous 90 days were significantly associated with MDRPA. In addition, ages 50-64 were associated with MDRPA when compared with ages 18-49 and while adjusting for covariates. No other factors were independently associated with MDRPA (Table 2).

\section{DISCUSSION}

The aim of this study was to identify factors associated with veterans with SCI/D with multidrug-resistant $P$. aeruginosa in a community setting. A quarter of this study population had community-associated $P$. aeruginosa-positive cultures. Our analysis showed that patients age 50-64 years have $80 \%$ greater odds of having a positive culture with MDRPA compared with the younger age group, whereas patients

Table 2 Multivariable GEE logistic regression analysis of risk factors for community-associated MDRPA

MDRPA vs nON-MDRPA

aOR $(95 \% \mathrm{Cl})$

$\begin{array}{ll}\text { Age (ref: 18-49 years) } & \\ 50-64 & 1.80(1.13-2.87) \\ \geqslant 65 \text { years } & 1.41(0.87-2.27) \\ & \\ \text { SCI/D level (ref: tetraplegia) } & \\ \text { Paraplegia } & 0.47(0.32-0.69) \\ \quad \text { Missing } & 1.54(0.90-2.65) \\ \text { Previous MDR } P \text {. aeruginosa culture in past } 365 \text { days } & 9.12(5.88-14.15) \\ \text { Carbapenems } & 2.56(1.35-4.87)\end{array}$

Abbreviations: aOR, adjusted odds ratio; $\mathrm{Cl}$, confidence interval; $\mathrm{GEE}$, generalized estimating equation; MDRPA, multidrug-resistant $P$. aeruginosa; MDR, multidrug-resistant; $\mathrm{SCl} / \mathrm{D}$, spinal cord injury and disorder. 
$\geqslant 65$ years had no significant association with MDRPA. These findings differ from other studies that found advanced age to be a risk for MDRPA and other infections. ${ }^{6}$

The study population of veterans with SCI/D contained patients with different levels of injury. When adjusting for other factors, a patient with paraplegia had 53\% lower odds of MDRPA compared with patients with tetraplegia. This is aligned with prior studies that found the level of injury influences the rate of infection. A patient with tetraplegia has greater functional impairments and thus a greater risk of infection. ${ }^{21}$

Previous MDRPA in the past year was a significant predictor of a positive MDRPA culture by more than nine times the odds of a non-MDRPA culture. Earlier studies have previously documented the recurrence of $P$. aeruginosa. A previous colonization or infection is associated with subsequent infections, with the pathogen commonly re-infecting the upper and lower respiratory tracts of patients. ${ }^{22,23}$ These existing strains are more often the cause of infection compared with exogenous sources. ${ }^{24}$ Relapse of $P$. aeruginosa may be due to an inappropriate initial antibiotic choice and duration of therapy. It also provides information regarding length of treatment for the new occurrence. $^{23}$

Along these lines, previous exposure to carbapenems increases the odds of MDRPA 2.56 times. Previous literature supports this association as a predictor for future resistant isolates. Exposure to these antibiotics can result in resistance through mechanisms including porin mutation, most commonly, and carbapenemase production. ${ }^{25-27}$ Because of the likelihood of reinfection of an existing strain, these mechanisms of resistance are important to understanding the risk of a previous carbapenem exposure.

This study had the advantage of longitudinal data from the Veterans' Health Administration, the largest integrated healthcare system in the US and largest provider of care for patients with SCI/D in the country. Using this data, our report is the largest known study of community-associated $P$. aeruginosa. However, because the data are collected from VA facilities, this study does not account for veterans who seek healthcare outside of the VA. This might introduce bias as there may be characteristics that are unique to that particular group of patients compared with this study population. Also, conclusions pertaining to veterans with SCI/D may not be generalizable to other populations with SCI/D because some of the injuries of these veterans may be related to their military service, which are unique circumstances. These Veterans also all receive care from the same healthcare system.

Another limitation of this study was the lack of differentiation between colonization and true infection with $P$. aeruginosa. Although MDRPA may be present in a culture, the patient may or may not have had systemic infection, and, depending on this assessment, may or may not have been prescribed antibiotic treatment. Therefore, whether prior MDRPA or non-MDRPA cultures were indicative of true infection or colonization may have impacted the prior antibiotic use variables and, thus, may have affected some of the associations observed in this study. Also, as previously mentioned, an assessment of whether MDRPA infection or colonization is recurrent or persistent is particularly important when examining $P$. aeruginosa epidemiology. This information could have helped us determine whether recurrent or persistent $P$. aeruginosa cultures were due to non-treatment, inadequate treatment, or a newly acquired strain from an exogenous source. However, to determine recurrence or persistence, identification of bacterial strain type is required. Although an approximation of strain similarity can be inferred from antibiotic susceptibility testing, definitive determination of bacterial strain type requires molecular testing such as pulsed-field gel electrophoresis or other genetic sequencing techniques. Unfortunately, this information was not available in the administrative data sets used for this study. Future studies examining the relationship between strain type and recurrent or persistent $P$. aeruginosa colonization and infection in patients with SCI/D will be helpful.

This study of veterans with SCI/D has identified that communityassociated MDRPA colonization and infection are associated with age, level of injury, prior MDRPA isolation and prior antibiotic exposure. Identification of these factors is important for the initiation of appropriate antibiotic therapy and the implementation of interventions to prevent the spread of MDRPA. As MDRPA is a serious threat per the Centers for Disease Control and Prevention, controlling its spread and acquisition in the community, as well as healthcare settings, is essential. These results should encourage research into effective prevention measures for community-associated multidrugresistant infections to protect this special population.

\section{DATA ARCHIVING}

There were no data to deposit.

\section{CONFLICT OF INTEREST}

The authors declare no conflict of interest.

\section{ACKNOWLEDGEMENTS}

We thank Scott Miskevics for his assistance with programming and Dr Beverly Gonzalez for her assistance with data analysis. This work was supported by The Department of Veterans Affairs, Veterans Health Administration, Office of Research and Development, Rehabilitation Research and Development Service Merit Review Award (B-1583-P), and Health Services Research and Development Service Post-Doctoral Fellowship Award (TPR 42-005). The views expressed in this article are those of the authors and do not necessarily reflect the position or policy of the Department of Veterans Affairs or the US government.

1 Centers for Disease Control and Prevention (US). Antibiotic resistance threats in the United States. 2013:69.

2 Sievert DM, Ricks P, Edwards JR, Schneider A, Patel J, Srinivasan A et al. Antimicrobial-resistant pathogens associated with healthcare-associated infections summary of data reported to the National Healthcare Safety Network at the Centers for Disease Control and Prevention, 2009-2010. Infect Control Hosp Epidemiol 2013; 34: 1-14.

3 Costa Pde O, Atta EH, Silva AR. Infection with multidrug-resistant gram-negative bacteria in a pediatric oncology intensive care unit: risk factors and outcomes. $J$ Pediatr (Rio J) 2015; 91: 435-441.

4 Boyle DP, Zembower TR. Epidemiology and management of emerging drug-resistant gram-negative bacteria: extended-spectrum $\beta$-lactamases and beyond. Urol Clin North Am 2015; 42: 493-505.

5 Aloush V, Navon-Venezia S, Seigman-Igra Y, Cabili S, Carmeli Y. Multidrug-resistant Pseudomonas aeruginosa: risk factors and clinical impact. Antimicrob Agents Chemother 2006; 50: 43-48.

6 Defez C, Fabbro-Peray P, Bouziges N, Gouby A, Mahamat A, Daures JP et al. Risk factors for multidrug-resistant Pseudomonas aeruginosa nosocomial infection. J Hosp Infect 2004; 57: 209-216.

7 Doi Y, Park YS, Rivera JI, Adams-Haduch JM, Hingwe A, Sordillo EM. Community-associated extended-spectrum $\beta$-lactamase-producing Escherichia coli infection in the United States. Clin Infect Dis 2013; 56: 641-648.

8 Tuon FF, Gortz LW, Rocha JL. Risk factors for pan-resistant Pseudomonas aeruginosa bacteremia and the adequacy of antibiotic therapy. Braz J Infect Dis 2012; 16: 351-356.

9 Cillóniz C, Gabarrús A, Ferrer M, Puig de la Bellacasa J, Rinaudo M, Mensa J et al. Community-acquired pneumonia due to multidrug and non-multidrug resistant Pseudomonas aeruginosa. Chest 2016; 150: 415-425.

10 Fujii A, Seki M, Higashiguchi M, Tachibana I, Kumanogoh A, Tomono K. Community-acquired, hospital-acquired, and healthcare-associated pneumonia caused by Pseudomonas aeruginosa. Respir Med Case Rep 2014; 12: 30-33. 
11 Hatchette TF, Gupta R, Marrie TJ. Pseudomonas aeruginosa community-acquired pneumonia in previously healthy adults: case report and review of the literature. Clin Infect Dis 2000; 31: 1349-1356.

12 Chang HT, Evans CT, Weaver FM, Burns SP, Parada JP. Etiology and outcomes of veterans with spinal cord injury and disorders hospitalized with community-acquired pneumonia. Arch Phys Med Rehabil 2005; 86: 262-267.

13 Evans CT, Burns SP, Chin A, Weaver FM, Hershow RC. Predictors and outcomes of antibiotic adequacy for bloodstream infections in veterans with spinal cord injury. Arch Phys Med Rehabil 2009; 90: 1364-1370.

14 Fitzpatrick MA, Suda KJ, Safdar N, Goldstein B, Jones MM, Poggensee L et al. Unique risks and clinical outcomes associated with extended-spectrum $\beta$-Lactamase enterobacteriaceae in veterans with spinal cord injury or disorder: a case-casecontrol study. Infect Control Hosp Epidemiol 2016; 37: 768-776.

15 Siroky MB. Pathogenesis of bacteriuria and infection in the spinal cord injured patient. Am J Med 2002; 113: 67S-79S.

16 Evans CT, LaVela SL, Weaver FM, Priebe M, Sandford P, Niemiec P et al. Epidemiology of hospital-acquired infections in veterans with spinal cord injury and disorder. Infect Control Hosp Epidemiol 2008; 29: 234-242.

17 Suda KJ, Patel UC, Sabzwari R, Cao L, Ramanathan S, Hill JN et al. Bacterial susceptibility patterns in patients with spinal cord injury and disorder (SCl/D): an opportunity for customized stewardship tools. Spinal Cord 2016; 54 1001-1009.

18 Magiorakos AP, Srinivasan A, Carey RB, Carmeli Y, Falagas ME, Giske CG et al. Multidrug-resistant, extensively drug-resistant and pandrug-resistant bacteria: an international expert proposal for interim standard definitions for acquired resistance. Clin Microbiol Infect 2012; 18: 268-281.
19 Charlson ME, Pompei P, Ales KL, MacKenzie CR. A new method of classifying prognostic comorbidity in longitudinal studies: development and validation. J Chronic Dis 1987; 40: 373-383.

20 Hanley JA, Negassa A, Edwardes MD, Forrester JE. Statistical analysis of correlated data using generalized estimating equations: an orientation. Am J Epidemiol 2003; 157: 364-375.

21 Montgomerie JZ. Infections in patients with spinal cord injuries. Clin Infect Dis 1997; 25: 1285-1290; quiz 1291-1292.

22 Harris A, Torres-Viera C, Venkataraman L, DeGirolami P, Samore M, Carmeli Y Epidemiology and clinical outcomes of patients with multiresistant Pseudomonas aeruginosa. Clin Infect Dis 1999; 28: 1128-1133.

23 Yum HK, Park IN, Shin BM, Choi SJ. Recurrent Pseudomonas aeruginosa infection in chronic lung diseases: relapse or reinfection? Tuberc Respir Dis (Seoul) 2014; 77: 172-177.

24 Talon D, Capellier G, Boillot A, Michel-Briand Y. Use of pulsed-field gel electrophoresis as an epidemiologic tool during an outbreak of Pseudomonas aeruginosa lung infections in an intensive care unit. Intensive Care Med 1995; 21: 996-1002.

25 Bubonja-Sonje M, Matovina M, Skrobonja I, Bedenic B, Abram M. Mechanisms of carbapenem resistance in multidrug-resistant clinical isolates of Pseudomonas aeruginosa from a Croatian hospital. Microb Drug Resist 2015; 21: 261-269.

26 Cavalcanti FL, Mirones CR, Paucar ER, Montes LÁ, Leal-Balbino TC, Morais MM et al. Mutational and acquired carbapenem resistance mechanisms in multidrug resistant Pseudomonas aeruginosa clinical isolates from Recife, Brazil. Mem Inst Oswaldo Cruz 2015; 110: 1003-1009.

27 Queenan AM, Bush K. Carbapenemases: the versatile beta-lactamases. Clin Microbiol Rev 2007; 20: 440-458. 